\title{
A BLIND RobUST WATERMARKING SCHEME BASED ON SVD AND CIRCULANT MATRICES
}

\author{
Noui Oussama ${ }^{1}$ and Noui Lemnouar ${ }^{2}$ \\ ${ }^{1}$ Department of Computer science, Faculty of Science University of Batna \\ os.noui@gmail.com \\ ${ }^{2}$ Department of Mathematics, Faculty of Science University of Batna \\ nouilemeyahoo.fr
}

\begin{abstract}
Multimedia security has been the aim point of considerable research activity because of its wide application area. The major technology to achieve copyright protection, content authentication, access control and multimedia security is watermarking which is the process of embedding data into a multimedia element such as image or audio, this embedded data can later be extracted from, or detected in the embedded element for different purposes. In this work, a blind watermarking algorithm based on SVD and circulant matrices has been presented. Every circulant matrix is associated with a matrix for which the SVD decomposition coincides with the spectral decomposition. This leads to improve the Chandra algorithm [1], our presentation will include a discussion on the data hiding capacity, watermark transparency and robustness against a wide range of common image processing attacks.
\end{abstract}

\section{KEYWORDS}

Digital image watermarking, Singular value Decomposition, circulant matrix, ownership protection.

\section{INTRODUCTION}

Due to improvements in the digital image technology and growing availability and usability of internet during the past several years, demands for storage and transmitting of digital images have seen a distinct increase, Unfortunately, the problem of illegal piracy is increasingly serious. Protection of digital multimedia content has become an increasingly important issue for content owners and service providers. Encryption data was a way to ensure only the owner to view the content, there are still ways for illegal using of the content after decryption $[2,3]$, that lead us to a new method for protection. Watermarking is the process of embedding data called a watermark into the multimedia object such that watermark can be detected or extracted later to make an assertion about the object. The object may be an audio, image or video and even 3-D models [4, 5]. Watermarking algorithms fall into two categories. The first form of watermarking was a spatial watermarking technique work with the pixel values directly. Generally, spatial domain watermarking is easy to implement from a computational point of view, but too fragile to resist numerous attacks $[6,7]$. In spatial domain, the watermark is directly embedded into the specific pixels of the host image, but in transform domain which our proposed scheme based in the watermark is embedded into the transform coefficients of the host image after applying DWT, DFT, DCT or SVD transform, and this called the frequency domain watermarking. Because of the

Dhinaharan Nagamalai et al. (Eds) : CSE, DBDM, CCNET, AIFL, SCOM, CICS, CSIP - 2014

pp. 65-77, 2014. (C) CS \& IT-CSCP 2014

DOI : $10.5121 /$ csit.2014.4406 
weakness of the spatial domain watermarks, watermarking in the frequency domain became more attractive as a result of its higher robustness against attacks compared to the spatial domain watermarking. To this aim a number of robust methods based on the SVD transform were introduced but these methods didn't offer good transparency and robustness against geometric attacks. Starting with Liu and Tan [8] an image watermarking method based on SVD, this method is robust against some attacks, and it is a non-blind method and it has a weak imperceptibility, Chandra et al. [1] also introduced a digital image watermarking method. This method is based on moderate modifying of the singular values of the host image. This method is weak against geometric attacks. Ganic et al. [9] proposed a method based on SVD in discrete wavelet transform (DWT) domain. The insertion procedure concerns the modifying of the singular values of the wavelet transformed sub-bands with the singular values of the mark. This scheme is a non-blind and the transparency of the watermarked image is weak. Makhloghi et al.[10] also proposed a scheme based on singular value decomposition in wavelet domain for copyright protection but his method lacks to robustness. Lin et al. [11] presented a full-band DWT domain image watermarking method based on SVD. This method has good robustness against common attacks but its drawback is that the original image is required in extraction procedure. Also the quality of the watermarked image is not good. The watermarking algorithms described in [1-8] are semi or non blind. Soumya Mukherjee and Arup Kumar Pal [12] proposed a robust watermarking scheme which employs both the Discrete Cosine Transform (DCT) and the Singular Value Decomposition (SVD), It starts with transformation of an original image into a transformed image using block based DCT. From each transformed block, the middle band DCT coefficients are selected to form a reduced transformed image and then the watermark is embedded into the constructed reduced transformed image after a suitable SVD operation. This method has good robustness against different attacks but it has a weak imperceptibility. In this paper, a new SVDbased method is proposed which gives the variety in creating the watermark under $(1,2, \ldots, \mathrm{n})$ blocks depending on the initiate coefficients and using the circulant's matrix properties to make Chandra algorithm [1] blind instead of non blind and turn it robust against different geometric and non geometric attacks. Organization of the paper is as follows: Section 2 explains the concept of SVD and Circulant matrices while Section 3 presents the proposed method. Section 4 throws light on the experimental results and a comparative analysis of our scheme and other schemes is given, whereas the summary of results and the conclusion is presented in Section 5.

\section{SINGULAR VALUE DECOMPOSITION AND CIRCULANT MATRICES}

\subsection{Singular value decomposition SVD}

Every real matrix $A$ can be decomposed into a product of three matrices :

$$
A=U \times S \times V^{t}
$$

where $U$ and $V$ are orthogonal matrices such that $U \times U^{t}=I$ and $V \times V^{t}=I$ where $I$ is the Identity matrix and $S$ is the diagonal matrix, $S=\operatorname{diag}\left(\partial_{1}, \partial_{2} \cdot \cdot\right)$ with $\partial_{1} \geq \partial_{2} \geq \cdot \cdot \geq 0$ The diagonal entries $\partial_{i}$ of $S$ are called the singular values of $A$, they are the eigenvalues of $A \times A^{t}$ or $A^{t} \times A$. The columns of $U$ are the left singular vectors of $A$, they are eigenvectors of $A \times A^{t}$ and the columns of $V$ called the right singular vectors of $A$ and they are eigenvectors of $A^{t} \times A$. 


\subsection{Circulant matrices}

The circulant matrix $C=\operatorname{cir}(c)$ associated to the vector $c \in R^{n}$ is the $n \times n$ matrix whose rows are given by iterations of the shift operator T acting on $\mathrm{c}$, its $K^{\text {th }}$ row is $T^{k} c, k=1, . ., n$ For example if $c=\left(c_{1}, c_{2}, c_{3}, c_{4}\right)$, the $4 \times 4$ circulant matrix

$$
C=\operatorname{cir}(c) \text { is giving by }\left(\begin{array}{llll}
c_{1} & c_{2} & c_{3} & c_{4} \\
c_{4} & c_{1} & c_{2} & c_{3} \\
c_{3} & c_{4} & c_{1} & c_{2} \\
c_{2} & c_{3} & c_{4} & c_{1}
\end{array}\right)
$$

\section{PROPOSED METHOD}

We consider a circulant matrix $c=\operatorname{cir}\left(c_{1}, c_{2}, c_{3}, c_{4}\right)$

The matrix $C C^{t}=C^{t} C$ is positive symmetric matrix so its spectral decomposition coincides with its SVD decomposition that is $C C^{t}=U_{0} \operatorname{diag}\left(\delta_{1}, \delta_{2}, \delta_{3}, \delta_{4}\right) U_{0}{ }^{t}$ with

$$
\begin{aligned}
& \delta_{1}=\left(c_{1}+c_{2}+c_{3}+c_{4}\right)^{2} \\
& \delta_{2}=\left(c_{1}-c_{2}+c_{3}-c_{4}\right)^{2} \\
& \delta_{3}=\delta_{4}=\left(c_{1}-c_{3}\right)^{2}+\left(c_{2}-c_{4}\right)^{2}
\end{aligned}
$$

are the singular values and $U_{0}$ is the constant matrix :

$$
U_{0}=\left(\begin{array}{cccc}
1 / 2 & -1 / 2 & 0 & -\sqrt{2} / 2 \\
1 / 2 & 1 / 2 & -\sqrt{2} / 2 & 0 \\
1 / 2 & -1 / 2 & 0 & \sqrt{2} / 2 \\
1 / 2 & 1 / 2 & \sqrt{2} / 2 & 0
\end{array}\right)
$$

If $A$ is an image of size $4 m \times 4 m$, to every vector $c=\left(c_{1}, c_{2}, c_{3}, c_{4}\right)$ is associated a $4 \times 4$ circulant matrix $C_{1}=\operatorname{cir}\left(c_{1}\right)$ and a watermark as $4 m \times 4 m$ matrix with one block

$$
W_{1}=\left(\begin{array}{cccc}
C_{1} C_{1}^{t} & 0 & \cdot & 0 \\
0 & 0 & & \cdot \\
\cdot & & \cdot & \cdot \\
0 & \cdot & \cdot & 0
\end{array}\right)
$$

To obtain a watermark $W_{k}$ with $k$ blocks 


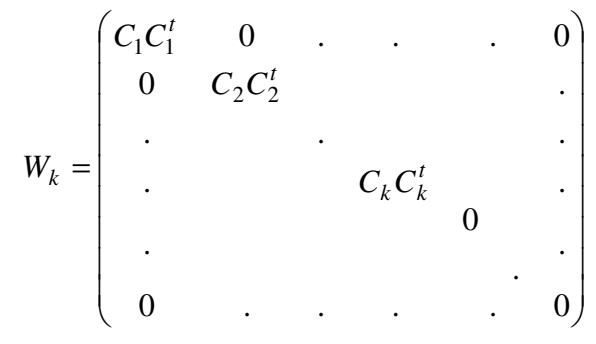

\subsection{Watermark insertion procedure}

To watermark a given original image $A$ of size $4 m \times 4 m$ we will use a watermark with one block as following:

1. Take $c=\left(c_{1}, c_{2}, c_{3}, c_{4}\right)$ such that $\partial_{4} \geq \partial_{3} \geq \partial_{2} \geq \partial_{1}$

2. Construct the watermark of size $4 m \times 4 m$

$$
W_{1}=\left(\begin{array}{cccc}
C_{1} C_{1}^{t} & 0 & . & 0 \\
0 & 0 & & . \\
. & & . & . \\
0 & . & . & 0
\end{array}\right)
$$

3. Apply SVD on $A$ :

$$
A=U \times S \times V^{t} \text { with } S=\operatorname{diag}\left(S_{i}\right)
$$

4. Apply SVD on $W_{1}$ :

$$
W_{1}=\left(\begin{array}{cc}
U_{0} & 0 \\
0 & I
\end{array}\right)\left(\begin{array}{ll}
\partial & 0 \\
0 & 0
\end{array}\right)\left(\begin{array}{cc}
U_{0}{ }^{t} & 0 \\
0 & I
\end{array}\right)
$$

With $\partial=\operatorname{diag}\left(\delta_{1}^{1}, \delta_{2}^{1}, \delta_{3}^{1}, \delta_{4}^{1}\right)$ and $I$ is $4(m-1) \times 4(m-1)$ identity matrix.

Put

$$
Y_{i}=S_{i}+\alpha \partial_{i}^{\prime}
$$

with $\partial_{1}^{\prime}=\delta_{1}^{1}, \partial_{2}^{\prime}=\delta_{2}^{1}, \partial_{3}^{\prime}=\delta_{3}^{1}, \partial_{4}^{\prime}=\delta_{4}^{1} \quad$ and $\forall i>4 \partial_{i}^{\prime}=0$

So

$$
A^{*}=U \times \operatorname{diag}\left(Y_{i}\right) \times V^{t}
$$

is the watermarked image.

\subsection{Watermarking detection and extraction procedure}

We don't require the original image $A$ to detect the watermark, we only require the watermarked image $A^{*}$, the scaling factor $\alpha$ and the key $S_{i}=\left(S_{1}, S_{2}, S_{3}, S_{4}\right)$ formed by the first four values of $S$. 
1. Apply SVD to $A^{*}$

2. Calculate

$$
A^{*}=U^{*} \times S^{*} \times V^{*^{t}}
$$

$$
x_{i}=\frac{S_{i}^{*}-S_{i}}{\alpha}
$$

for the first four elements.

If $x_{3}=x_{4}$ then the mark is detected else the watermark is not present on the image.

To extract the mark we compute:

$$
W^{*}=\left(\begin{array}{cc}
U_{0} & 0 \\
0 & I
\end{array}\right)\left(\begin{array}{cc}
X & 0 \\
0 & 0
\end{array}\right)\left(\begin{array}{cc}
U_{0}{ }^{t} & 0 \\
0 & I
\end{array}\right)
$$

where $X=\operatorname{diag}\left(x_{1}, x_{2}, x_{3}, x_{4}\right)$ and $I$ is $4(m-1) \times 4(m-1)$ identity matrix.

\section{Remarks:}

1. If we use a watermark $W_{k}$ with $k$ blocks, to detect or extract the watermark we only require the scaling factor $\alpha$ and a key $K_{2}=\left(S_{1}, \ldots, S_{4 k}\right)$ of length $4 \mathrm{k}$ which contains the first $4 \mathrm{k}$ values of $\mathrm{S}$. In this case the sequence $\mathrm{X}=\left(x_{i}\right)$ is of length $4 \mathrm{k}$ and the mark is detected if $x_{4 i-1}=x_{4 i}$ for $i=1, \ldots, k$.

2. In Chandra algorithm [1], to extract the watermark (W), $U_{w}, V_{w}$ are required, in the proposed scheme $U_{w}=V_{w}$ is a constant matrix and independent of the watermark, thus our proposed algorithm is blind.
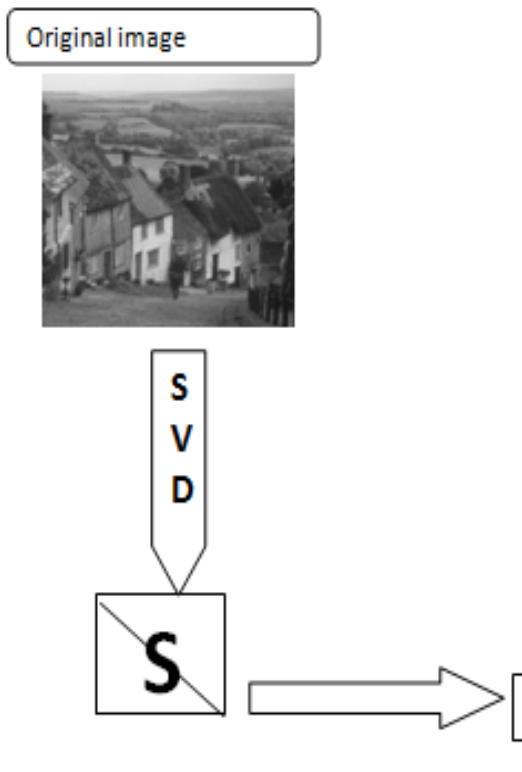

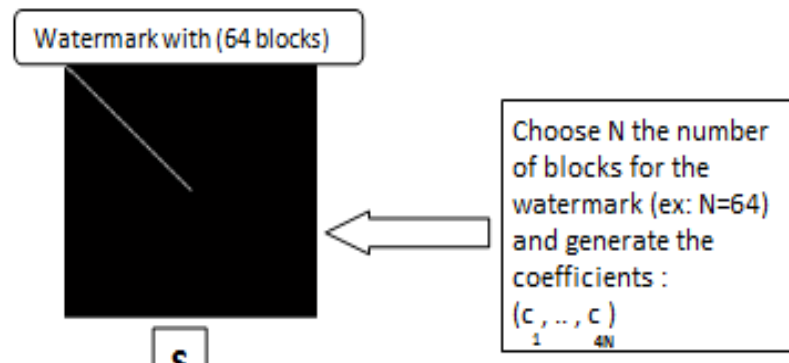

\section{s}

V

D
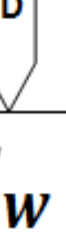

Embedding the watermark
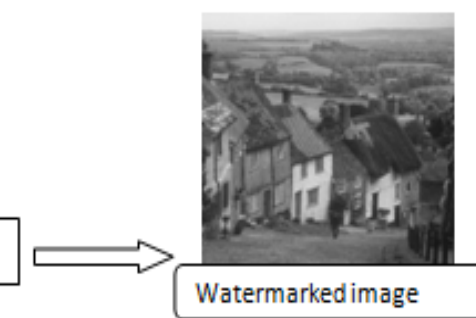

Figure 1. The Proposed watermarking scheme.

Our proposed watermarking method can be concluded in (Figure 1). As it is shown to construct the watermark we first choose $\mathrm{N}$ the number of blocks of the watermark then we generate the 
coefficients $\left(c_{1}, . ., c_{4 N}\right)$ and by following the steps mentioned above we embed the watermark into the original image.

\section{EXPERIMENTAL RESULTS}

The proposed scheme is implemented using MATLAB. Six $512 \times 512$ images Lena, Goldhill, Baboon, Barbara, Peppers, Boat were used in the simulation (figure 2). The signature is a $512 \times$ 512 image composed with $\mathrm{N}$ blocks in its diagonal.
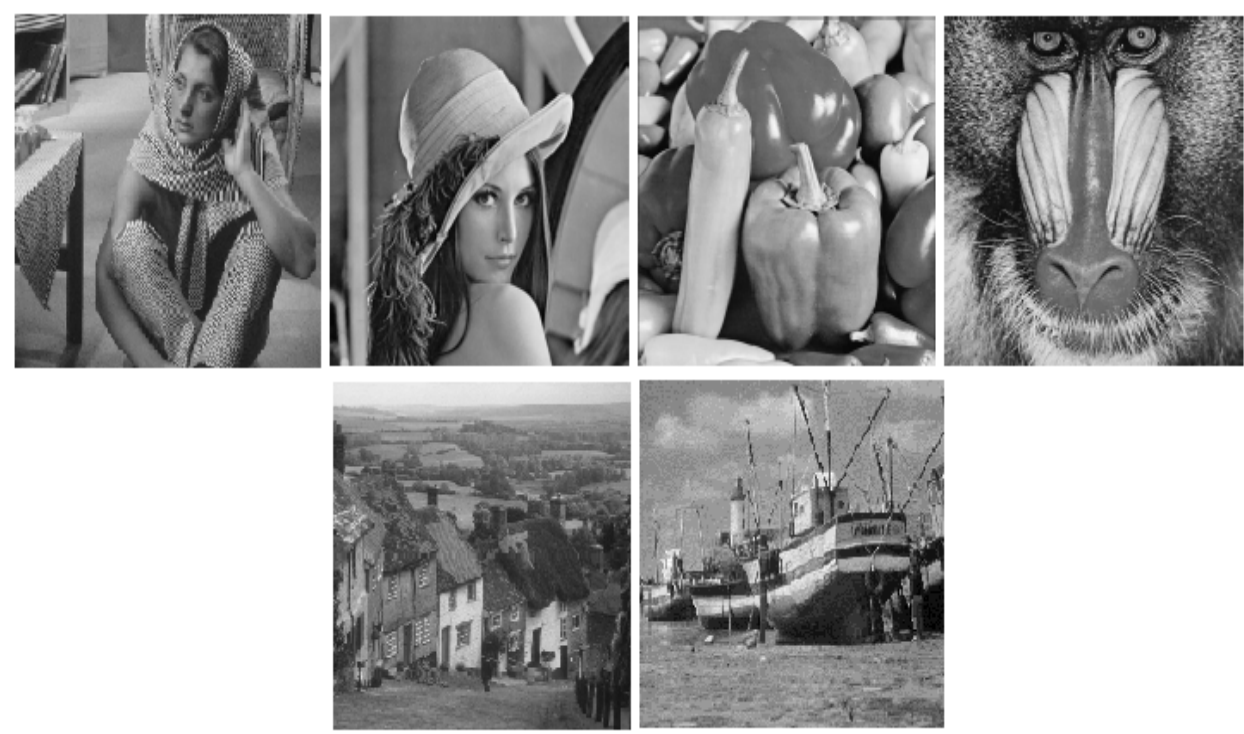

Figure 2. Original test images

To evaluate the quality of the watermarked images we use the PSNR measure defined as:

$$
P S N R=10 \log _{10}\left(\frac{255^{2}}{M S E}\right) d b
$$

With MSE is the mean squared error between the original and watermarked image. The PSNR values of the watermarked images by our method indicate that our method in general achieves very good quality as it shown in (Table 1).

Table 1. Relationship of number of blocks of the watermark and PSNR of different watermarked images.

(dB) Sf sets to 0.06

\begin{tabular}{|l|l|l|l|l|l|l|l|l|l|}
\hline Image name Nb blocks & 1 & 3 & 5 & 10 & 30 & 64 & 80 & 100 & 128 \\
\hline Lena & 56.6994 & 55.5982 & 55.7382 & 55.0831 & 55.8089 & 56.0630 & 55.4090 & 55.4366 & 55.3805 \\
\hline Goldhill & 57.2825 & 55.5499 & 55.4955 & 55.1460 & 54.6665 & 54.6762 & 52.9705 & 52.8710 & 52.8454 \\
\hline Baboon & 55.6458 & 54.6902 & 55.0781 & 55.1058 & 51.6568 & 50.4369 & 49.4199 & 49.1576 & 49.2020 \\
\hline Barbara & 56.9139 & 55.5102 & 57.1737 & 55.2064 & 53.3403 & 51.5035 & 50.2628 & 50.1065 & 50.0764 \\
\hline Peppers & 55.9094 & 55.2634 & 55.1153 & 53.5932 & 54.5946 & 55.4747 & 56.2183 & 56.2190 & 56.2759 \\
\hline Boat & 57.1737 & 55.6458 & 55.2064 & 54.6902 & 51.5035 & 52.9705 & 52.8710 & 52.8454 & 52.8710 \\
\hline
\end{tabular}

Its clear from (Table1) that the proposed method preserves good transparency for the watermarked images. 


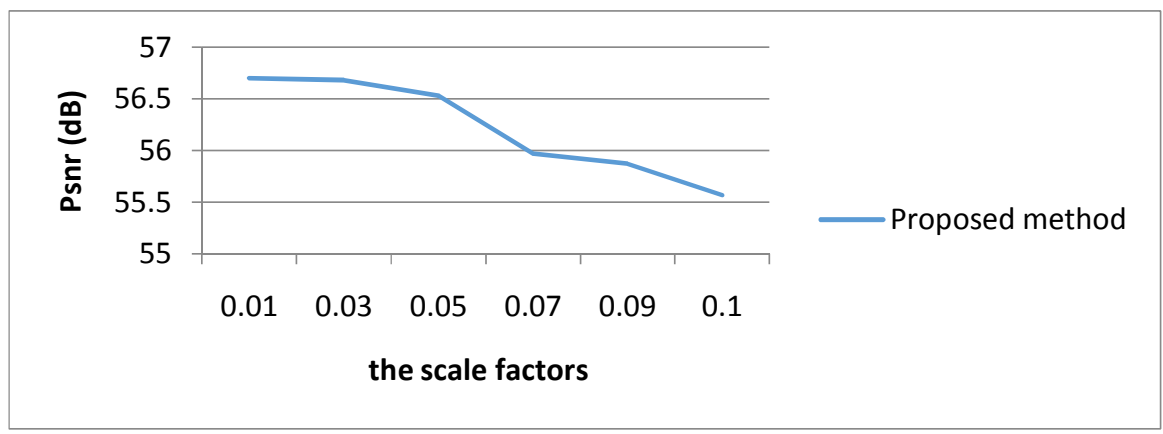

Figure 3. Relationship of the scale factors $(\alpha)$ and PSNR for the proposed method

(Figure 3) shows a relation between $\alpha$ and transparency in terms of the PSNR value. We can notice that our method has a good PNSR values and it reach its optimal value when the scale factors $(\alpha)$ is between 0.01 and 0.03 .

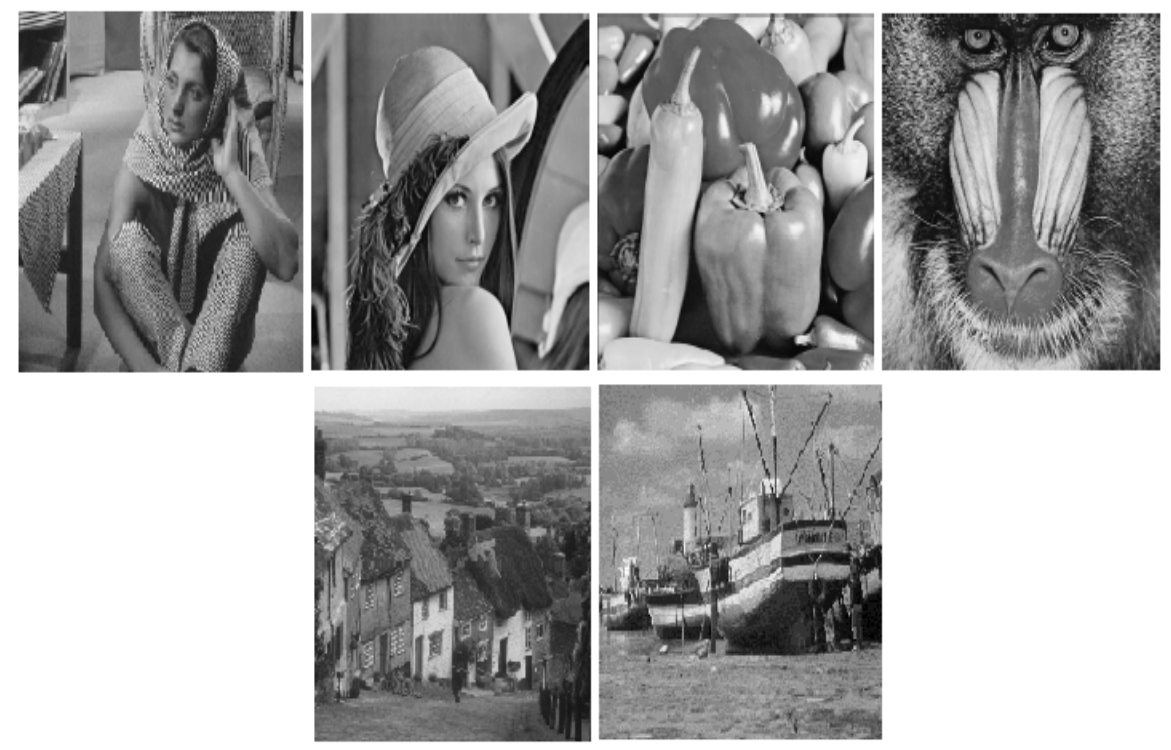

Figure 4. Watermarked images

(Figure 4) shows the watermarked images used in the simulation, it can be seen that there is no perceptual difference between original and watermarked images that is also supported by good PSNR value. It shows that the good imperceptibility is obtained by proposed technique.

We use normalized correlation (NC) to evaluate the quality of the extracted watermark, and this measure is defined by:

$$
N C\left(W, W^{\prime}\right)=\frac{1}{W_{h} \times W_{w}} \sum_{i=0}^{W_{h}-1} \sum_{j=0}^{W_{W-1}} W(i, j) \times W^{\prime}(i, j)
$$

with $W_{h}$ and $W_{w}$ are the height and width of the watermarked image ,respectively. $W(i, j)$ and $W^{\prime}(i, j)$ denote the coefficients of the inserted watermark and the extracted watermark respectively. 
As we can see in (Table 2) our proposed method is robust against different geometric and nongeometric attacks, and we can notice also that the relationship between the number of blocks of the watermark and the resulted $N C$ generally are inversely proportional except for the rotation attack.

Table 2. Resulted NC for different image attacks using variation of watermarks.

\begin{tabular}{|c|c|c|c|c|c|c|c|c|c|c|c|c|c|}
\hline \multirow{2}{*}{ Attacks } & \multicolumn{3}{|c|}{ Jpeg } & \multirow{2}{*}{$\begin{array}{l}\text { speckle } \\
0.04\end{array}$} & \multirow[t]{2}{*}{ imsharpen } & \multirow[t]{2}{*}{ Smooth } & \multicolumn{2}{|c|}{ Rotation } & \multirow{2}{*}{$\begin{array}{l}\text { salt \& } \\
\text { pepper }\end{array}$} & \multirow[t]{2}{*}{ FFT } & \multirow{2}{*}{$\begin{array}{l}\text { Filtre } \\
\text { median }\end{array}$} & \multirow{2}{*}{$\begin{array}{l}\text { translate } \\
\text { [20 35] }\end{array}$} & \multirow{2}{*}{\begin{tabular}{|l|}
$\begin{array}{l}\text { Gaussian } \\
\text { filter }\end{array}$ \\
hsize $=[55]$ \\
sigma $=2$
\end{tabular}} \\
\hline & 50 & 60 & 90 & & & & $3^{\circ}$ & $5^{\circ}$ & & & & & \\
\hline 1 & 0.9941 & 0.9941 & 0.9941 & 0.9985 & 0.9996 & 0.9993 & 0.9684 & 0.8477 & 0.9998 & 0.9941 & 1.0000 & 0.9989 & 0.9994 \\
\hline 3 & 0.9577 & 0.9577 & 0.9578 & 0.9952 & 0.9982 & 0.9982 & 0.9517 & 0.8560 & 0.9997 & 0.9581 & 0.9998 & 0.9941 & 0.9974 \\
\hline 5 & 0.9236 & 0.9236 & 0.9238 & 0.9928 & 0.9972 & 0.9975 & 0.9471 & 0.8634 & 0.9996 & 0.9246 & 0.9993 & 0.9906 & 0.9947 \\
\hline 10 & 0.8596 & 0.8598 & 0.8603 & 0.9900 & 0.9931 & 0.9941 & 0.9454 & 0.8822 & 0.9993 & 0.8623 & 0.9962 & 0.9882 & 0.9756 \\
\hline 30 & 0.7551 & 0.7559 & 0.7584 & 0.9885 & 0.9830 & 0.9489 & 0.9582 & 0.9195 & 0.9985 & 0.7656 & 0.9615 & 0.9889 & 0.8280 \\
\hline 64 & 0.7235 & 0.7249 & 0.7280 & 0.9867 & 0.9796 & 0.8864 & 0.9711 & 0.9423 & 0.9978 & 0.7373 & 0.9226 & 0.9911 & 0.7058 \\
\hline 80 & 0.7102 & 0.7123 & 0.7143 & 0.9825 & 0.9782 & 0.8459 & 0.9740 & 0.9465 & 0.9961 & 0.7243 & 0.8979 & 0.9927 & 0.6360 \\
\hline 100 & 0.7082 & 0.7107 & 0.7121 & 0.9807 & 0.9781 & 0.8389 & 0.9755 & 0.9487 & 0.9952 & 0.7220 & 0.8939 & 0.9929 & 0.6243 \\
\hline 128 & 0.7072 & 0.7100 & 0.7115 & 0.9803 & 0.9781 & 0.8371 & 0.9757 & 0.9491 & 0.9945 & 0.7213 & 0.8924 & 0.9928 & 0.6214 \\
\hline
\end{tabular}

Table 3. Comparison of PSNR for Lai et al. [13], Tsai et al [14] and our algorithm.

\begin{tabular}{|l|ccccc|}
\hline \multirow{2}{*}{ Method } & \multicolumn{5}{|c|}{ the scale factors } \\
\cline { 2 - 6 } & 0.01 & 0.03 & 0.05 & 0.07 & 0.09 \\
\hline Lai et al .[13] & 51.14 & 51.14 & 50.89 & 49.52 & 47.49 \\
\hline Tsai et al [14] & 47 & 37 & 33 & 28 & about 25 \\
\hline Proposed method & 56.70 & 56.68 & 56.53 & 55.97 & 55.87 \\
\hline
\end{tabular}

(Table 3) shows the comparison of PSNR for two other algorithms and our algorithm. The values of the scale factors, (SFs) are carried out with constant range from 0.01 to 0.09 with an interval of 0.02 . Size of host images are $256 \times 256$ for Lai et al. [13], and $512 \times 512$ for Tsai et al. [14] and our scheme.

Table 4. The comparison of robustness and imperceptibility (dB) for our scheme and Soumya et al. [12] under various image processing attacks.

\begin{tabular}{|l|l|l|l|l|}
\hline \multicolumn{1}{|c|}{ Attack } & $\begin{array}{l}\text { NC of the } \\
\text { extracted } \\
\text { watermark } \\
\text { (Proposed } \\
\text { Scheme) }\end{array}$ & $\begin{array}{l}\text { PSNR of the } \\
\text { Attacked } \\
\text { watermarked } \\
\text { image } \\
\text { (Proposed } \\
\text { Scheme) }\end{array}$ & $\begin{array}{l}\text { NC of the } \\
\text { extracted } \\
\text { watermark } \\
{[12]}\end{array}$ & $\begin{array}{l}\text { PSNR of the } \\
\text { Attacked } \\
\text { watermarked } \\
\text { image [12] }\end{array}$ \\
\hline $\begin{array}{l}\text { Gaussian Lowpass } \\
\text { Filtering (3 } \times 3)\end{array}$ & $\mathbf{0 . 9 9 9 1}$ & $\mathbf{5 6 . 1 7 3 4 ~ d B}$ & 0.9974 & $41.2799 \mathrm{~dB}$ \\
\hline Average Filtering & $\mathbf{0 . 9 9 4 6}$ & $\mathbf{5 2 . 6 9 8 0 ~ d B}$ & 0.8253 & $35.7368 \mathrm{~dB}$ \\
\hline $\begin{array}{l}\text { Image Noising by salt- } \\
\text { and-pepper noise }\end{array}$ & $\mathbf{0 . 9 9 2 7}$ & $\mathbf{5 0 . 1 4 1 3 ~ d B}$ & 0.9009 & $24.7876 \mathrm{~dB}$ \\
\hline
\end{tabular}




\begin{tabular}{|c|c|c|c|c|}
\hline $\begin{array}{l}\text { image enhancement by } \\
\text { histogram } \\
\text { equalization }\end{array}$ & 0.9122 & $40.0745 \mathrm{~dB}$ & 0.9254 & $12.1774 \mathrm{~dB}$ \\
\hline $\begin{array}{l}\text { Center-cropped attack } \\
(64 \times 64 \text { pixels }) \\
\text { and filled with pixel value } \\
0\end{array}$ & 0.9178 & $41.4920 \mathrm{~dB}$ & 0.9417 & $20.8188 \mathrm{~dB}$ \\
\hline $\begin{array}{l}\text { Center-cropped attack } \\
(64 \times 64 \text { pixels }) \\
\text { and filled with pixel value } \\
255\end{array}$ & 0.9582 & $24.7561 \mathrm{~dB}$ & 0.8983 & $15.0842 \mathrm{~dB}$ \\
\hline $\begin{array}{l}\text { Center-cropped attack } \\
(128 \times 128 \\
\text { pixels }) \text { and filled with } \\
\text { pixel value } 0\end{array}$ & 0.8793 & $26.3998 \mathrm{~dB}$ & 0.8979 & $15.3449 \mathrm{~dB}$ \\
\hline $\begin{array}{l}\text { Center-cropped attack } \\
(128 \times 128 \\
\text { pixels }) \text { and filled with } \\
\text { pixel value } 255\end{array}$ & 0.9577 & $13.8987 \mathrm{~dB}$ & 0.8743 & $8.8648 \mathrm{~dB}$ \\
\hline $\begin{array}{l}\text { JPEG Compression } \\
(\mathrm{QF}=25)\end{array}$ & 0.9979 & $55.4146 \mathrm{~dB}$ & 0.9281 & $35.9800 \mathrm{~dB}$ \\
\hline $\begin{array}{l}\text { JPEG Compression } \\
(\mathrm{QF}=50)\end{array}$ & 0.9979 & $55.4132 \mathrm{~dB}$ & 1 & $38.7407 \mathrm{~dB}$ \\
\hline
\end{tabular}

The bold values indicates the best values comparing with the others.

(Table 4) presents the comparison of robustness and imperceptibility $(\mathrm{dB})$ for our scheme and Soumya et al. [12] under various image processing attacks, besides the robustness results the proposed scheme achieved high imperceptibility compared with Soumya et al. [12 


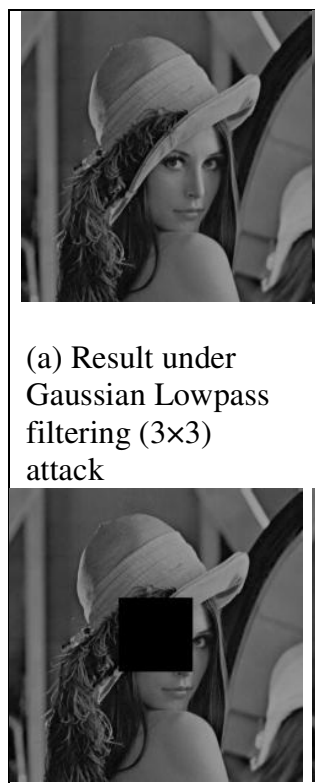

(e) Result under Center-cropped attack $(64 \times 64$ pixels) and filled with pixel value 0 (b) Result under Average filtering attack

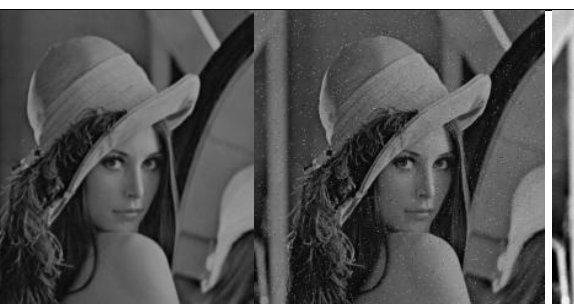

(c) Result under salt-and-pepper noise attack

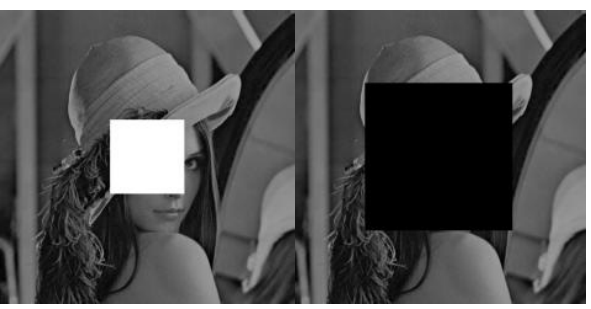

(f) Result under Center-cropped attack $(64 \times 64$ pixels) and filled with pixel value 255 (g) Result under Center-cropped attack $(128 \times 128$ pixels) and filled with pixel value 0

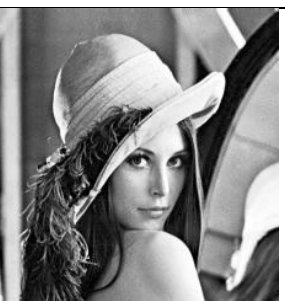

(d) Result under histogram equalization attack

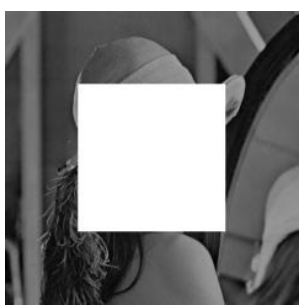

(h) Result under Center-cropped attack $(128 \times 128$ pixels) and filled with pixel value 255

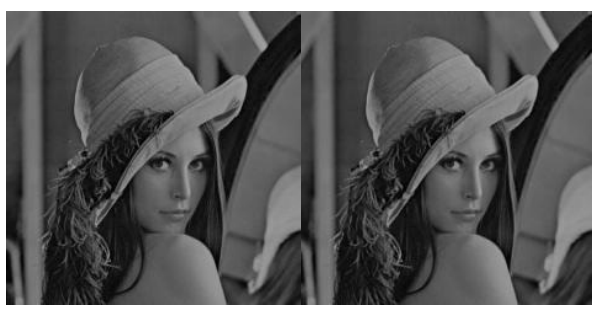

(i) Result under JPEG Compression

(j) Result under $(\mathrm{QF}=50)$ attack JPEG Compression $(\mathrm{QF}=25)$ attack

Figure 5. The attacked watermarked image under various image processing attacks in the comparison with Soumya et al. [12]

(Figure 5) shows the attacked images of the comparison with the scheme of Soumya et al.[12]. 


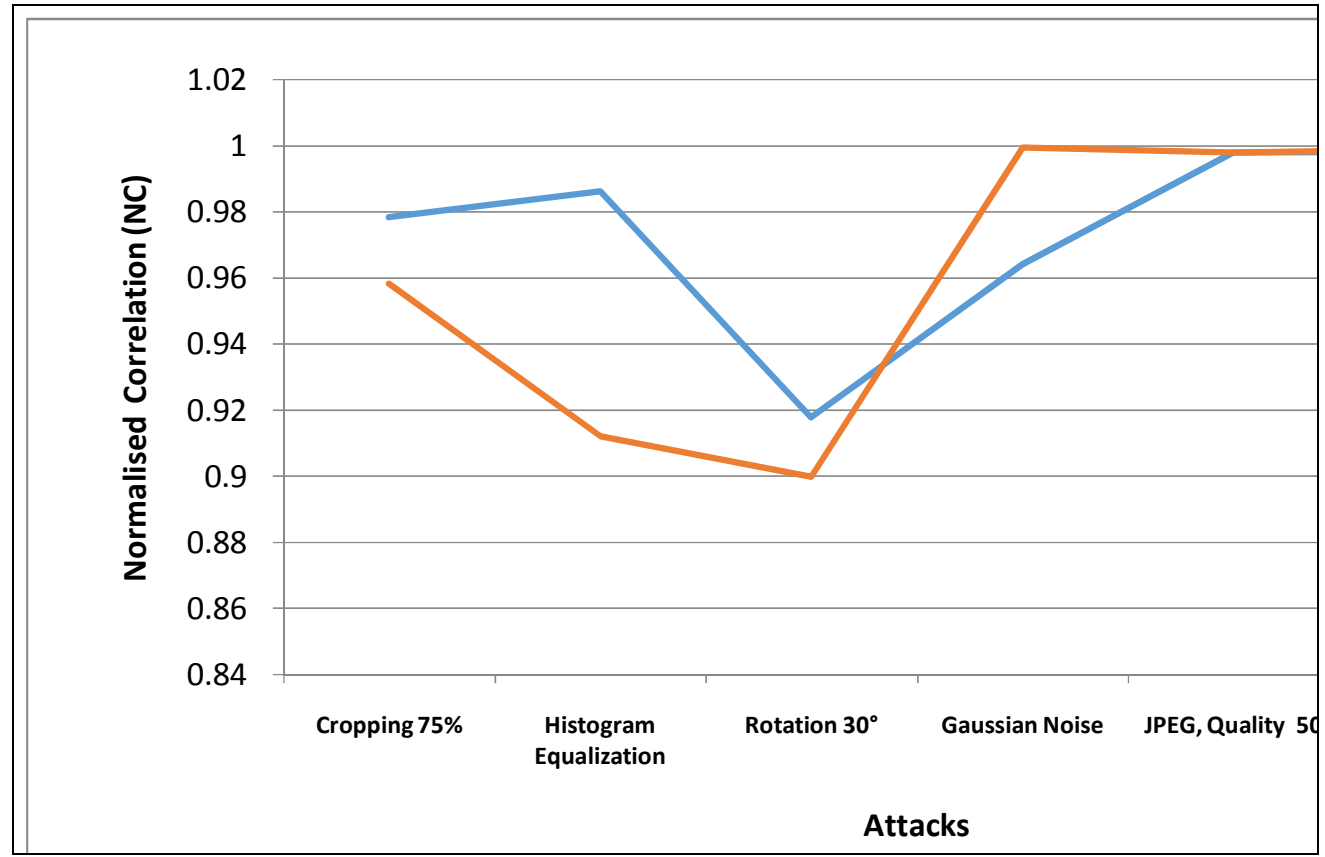

Figure 6. Comparison of our algorithm and Musrat et al [15] in term of $N C$ values

(Figure 6) presents a comparison of robustness between the proposed method and Musrat et al [15] method, it shows that Musrat's scheme is more robust against such attacks as cropping $75 \%$, histogram equalization and rotation $30^{\circ}$ compared to our method but ours still achieves a good $\mathrm{NC}$ values after applying those attacks, and the minimum value of $\mathrm{NC}$ was 0.9 which means its robust, and for the other attacks (Gaussian noise, JPEG compression and Translation) our method perform better than Musrat's scheme.

Table 5. The comparison of robustness for our scheme, Nasrin et al [17], Lai et al. [13] and Rastegar et al. [16].

\begin{tabular}{|c|c|c|c|c|c|}
\hline Attack & $\begin{array}{l}\text { Proposed } \\
\text { scheme }\end{array}$ & Nasrin et al [17] & Lai et al .[13] & $\begin{array}{l}\text { Rastegar et } \\
\text { al.[16] }^{a}\end{array}$ & $\begin{array}{l}\text { Rastegar } \\
\text { et al.[16] }\end{array}$ \\
\hline Pepper \& salt noise $(0.3)$ & 0.9927 & 0.8926 & - & 0.7515 & 0.8258 \\
\hline Speckle noise $($ var $=0.01)$ & 0.9950 & 0.952 & - & 0.9609 & 0.9667 \\
\hline Gaussian noise $(\mathrm{M}=0, \mathrm{var}=0.5)$ & 0.9210 & 0.8935 & - & 0.7926 & 0.82 \\
\hline Gaussian filtering $(3 \times 3)$ & 0.9990 & 0.987 & - & 0.8023 & 0.9843 \\
\hline Median filtering $(3 \times 3)$ & 0.9885 & 0.982 & 0.9597 & 0.7534 & 0.9706 \\
\hline Wiener filtering $(3 \times 3)$ & 0.9826 & 0.984 & - & 0.9824 & 0.9569 \\
\hline Sharpening & 0.9966 & 0.932 & - & 0.9687 & 0.9511 \\
\hline Histogram equalization & 0.9122 & 0.990 & 0.9862 & 0.9648 & 0.9628 \\
\hline Gamma correction $(0.7)$ & 0.9887 & 0.9935 & 0.9982 & - & - \\
\hline Gamma correction $(0.8)$ & 0.9890 & 0.9950 & - & 0.7203 & 0.9217 \\
\hline JPEG compression $Q=30$ & 0.9937 & 0.987 & - & - & - \\
\hline JPEG compression $Q=10$ & 0.9915 & 0.972 & 0.9772 & 0.9824 & 0.9843 \\
\hline JPEG compression $Q=5$ & 0.9907 & 0.952 & - & 0.8532 & 0.9354 \\
\hline Scaling zoom $($ out $=0.5$, in $=2)$ & 0.9772 & 0.948 & - & 0.5127 & 0.953 \\
\hline Rotation $\left(\right.$ angle $\left.=2^{\circ}\right)$ & 0.9648 & 0.981 & - & 0.5068 & 0.9628 \\
\hline Rotation $\left(\right.$ angle $\left.=-30^{\circ}\right)$ & 0.8532 & 0.9823 & 0.9780 & - & - \\
\hline
\end{tabular}

' - ' means the attacks are not done.

The bold values indicates the best values comparing with the others.

a Indicates the first scheme in Rastegar et al. [16]

b Indicates the second scheme in Rastegar et al.[16] 
(Table 5) shows the proposed results with Rastegar's schemes results and Lai's scheme results and Nasrin et al's scheme when scaling factor is 0.05. Rastegar scheme (a) represents the embedding in all sub-bands while Rastegar scheme (b) represents the embedding in LH and HL only. Our scheme performed better than Nasrin's , Lai's and Rastegar's schemes as shown in (Table 5) .

\section{CONCLUSIONS}

This paper presents a blind robust digital image watermarking scheme based on singular value decomposition and on circulant matrix for copyright protection, using the circulant matrix's properties we improved the algorithm of Chandra and turned it to a blind watermarking algorithm after it was a non blind in addition to the augmentation of its robustness. Simulation results indicate that the proposed method achieves higher robustness compared to other known watermarking methods. The proposed method is robust against a wide range of common image processing attacks.

\section{REFERENCES}

[1] D. S. Chandra, "Digital image watermarking using singular value decomposition," Proceedings of the 45th mid-west Symposium on Circuits ，Systems (MndWSCAS'02), vol.3, 2002, pp.264-267.

[2] B. WR,D.Gruhl,N.Morimoto,A.Lu,Techniques for data hiding ,IBM Systems Journa 135(1996)313336.

[3] C.S. Lu,Multimedia Security :Stenography and Digital Watermarking Techniques for Protection of Intellectual Property ,PA Pub: Idea Group Pub,Hershey157-172.

[4] M.D.Swanson,M.KabayashiA.H.Tewfik, Multimedia data embedding and watermarking technologies ,in:IEEE,vol.86,no.6, June 1998,pp.1064-1087.

[5] F.Hartung M. Kutter, Multimedia watermarking techniques,in: IEEE, vol. 87, no.7,July1999,pp.10791107 .

[6] W. H. Lin, Y. R. Wang, and S. J. Horng, "A Blind Watermarking Scheme Based on Wavelet Tree Quantization," The Second International Conference on Secure System Integration and Reliability Improvement, 2008, pp. 89-94.

[7] M. Ouhsain and A. B. Hamza, " Image watermarking scheme using non-negative matrix factorization and wavelet transform," Journal of Expert Systems with Applications, vol. 36, pp. 2123-2129, 2009.

[8] R. Liu and T. Tan, "A SVD-based watermarking scheme for protecting rightful ownership," IEEE Transactions on Multimedia, vol. 4, no. 1, pp. 121-128, 2002.

[9] E. Ganic and A. M. Eskicioglu, "Robust DWT-SVD domain image watermarking: embedding data in all frequencies," ACM Multimedia and Security Workshop 2004, Germany, 2004, pp. 20-21.

[10] Makhloghi, M.; Akhlaghian, F.; Danyali, H., "Robust digital image watermarking using singular value decomposition," Signal Processing and Information Technology (ISSPIT), 2010 IEEE International Symposium on , vol., no., pp.219,224, 15-18 Dec. 2010

[11] C.H. Lin, J.C. Liu, and P.C. Han, "On the security of the full-band image watermark for copyright protection," IEEE International Conference on Sensor Networks, Ubiquitous, and Trustworthy Computing, 2008, pp. 74-79.

[12] Soumya Mukherjee and Arup Kumar Pal. 2012. A DCT-SVD based robust watermarking scheme for grayscale image. In Proceedings of the International Conference on Advances in Computing, Communications and Informatics (ICACCI '12). ACM, New York, NY, USA, 573-578.

[13] C. Lai and C. Tsai, "Digital Image Watermarking Using Discrete Wavelet Transform and Singular Value Decomposition," IEEE Transactions on Instrumentation and Measurement, vol. 59, no. 11, November 2010.

[14] H.-hsu Tsai, Y.-jie Jhuang, and Y.-shou Lai, "An SVD-based image watermarking in wavelet domain using SVR and PSO,” Applied Soft Computing Journal, vol. 12, no. 8, pp. 2442-2453, 2012.

[15] Musrrat Ali and Chang Wook Ahn. 2013. An optimized watermarking technique employing SVD in DWT domain. In Proceedings of the 7th International Conference on Ubiquitous Information Management and Communication (ICUIMC '13). ACM, New York, NY, USA, , Article 86, 7 page 
[16] Rastegar S, Namazi F, Yaghmaie K, Aliabadian A. Hybrid watermarking algorithm based on singular value decomposition and radon transform. Int J Electron Commun (AEU) 2011;65(7):658-63.

[17] Nasrin M. Makbol, Bee Ee Khoo, Robust blind image watermarking scheme based on Redundant Discrete Wavelet Transform and Singular Value Decomposition, AEU - International Journal of Electronics and Communications, Volume 67, Issue 2, February 2013, Pages 102-112, ISSN 1434841 